\title{
Arbor
}

\section{Financiación y futuro, claves para un equilibrio}

\section{Rafael Sariego García}

Arbor CLXXX, 710 (Febrero 2005), 353-361 pp.

\section{Introducción}

Es indudable que, en las últimas semanas, ha cobrado más presencia si cabe en el debate social el problema de la financiación sanitaria. En un claro ejercicio de responsabilidad, deberíamos realizar el esfuerzo de, al menos, no instrumentalizar ni convertir este asunto en un arma arrojadiza o elemento que perpetúe situaciones de confrontación que, en nada enriquecen el debate y ningún valor añadido suponen al Sistema Nacional de Salud, que doy por supuesto todos defendemos. Hemos observado como en los últimos meses, cuestiones vinculadas a la financiación del SNS se han levantado como el principal protagonista de una situación en la que, si queremos llegar a un resultado óptimo, debemos imprimir la calma y la serenidad como instrumentos de trabajo para un futuro a corto plazo. El problema es real y la necesidad de aportar soluciones es evidente, pero desde Asturias no permitiremos que se puedan cerrar a cualquier precio.

Desde el Gobierno del Principado de Asturias entendemos que el SNS es un patrimonio colectivo generado con el esfuerzo de todos los españoles. Un patrimonio irrenunciable con el que tenemos contraída la obligación de garantizar su permanencia en el tiempo con sólidas bases de futuro, de manera que las futuras generaciones puedan seguir disfrutando del mismo servicio público que hoy tenemos a nuestra disposición. Hemos adquirido ante nuestros ciudadanos el compromiso de asegurar la sostenibilidad del sistema, en la parte que nos corresponde, y que nos permita ofrecer una atención sanitaria en igualdad de condiciones que el resto del país. Esta es la esencia del llamado Estado de Bienestar, una condi- 


\section{Rafael Sariego García}

ción a la que accedemos gracias al esfuerzo de todos, y que nos podemos consentir que se quiebre por intereses ajenos a la defensa del equilibrio del SNS.

En ese sentido, hace algo mas de dos años asumimos por transferencia la responsabilidad de la asistencia sanitaria en Asturias, en la convicción de que éramos capaces de contribuir a la mejora de nuestro sistema público. DE forma simultánea, entró en vigor el nuevo modelo de financiación autonómica, que incluyó la financiación sanitaria en la financiación general de las comunidades autónomas.

Cuando aún no han transcurrido tres años de la puesta en marcha del nuevo modelo, se ha puesto de manifiesto que, en la mayoría de los casos, el nivel de crecimiento de los gastos mantiene un ritmo superior al de los ingresos. Así se origina un déficit que, a medio plazo, pone en cuestión la sostenibilidad financiera del sistema y en peligro el desarrollo de políticas de progreso dirigidas a los usuarios.

No es menos cierto y, así debemos aceptarlo en aras del rigor del debate, que el uso de los recursos disponibles también debe ser sometido al correspondiente análisis con objeto de superar las posibles causas de ineficiencia en el gasto. En Asturias somos corresponsables con esta situación, y hemos asumidos medidas como la aplicación del recargo autonómicos del impuesto sobre los hidrocarburos, el llamado céntimo sanitario, para dar un ejemplo de que nos enfrentamos al problema de forma directa, y evitando los rodeos. Aceptamos el coste social para nuestros administrados, pero también entendemos que, de no ejercer estas fórmulas que a nadie gustan, luego es difícil sostener un discurso reivindicativo. Es decir, resulta sencillo pedir si antes no se han utilizado todos los recursos a tu alcance, pero este Gobierno tiene claro su responsabilidad en el conjunto del SNS. Estamos en condiciones morales y políticas de exigir el mismo trato que a los demás, pero quizá también en un escalón superior porque hemos puesto en marcha todos los mecanismos a nuestro alcance para paliar el déficit.

También parece evidente que los cambios demográficos, consecuencia de los movimientos poblacionales o de la estructura de la población (envejecimiento, en el caso de algunas comunidades) deben ser objeto de valoración, pero nunca argumento prioritario para un método que establezca estas pautas como traducción del problema o síntesis de la solución. Asturias se opone al pago por población, ya que rompería el equilibrio del sistema, y lo que pide es que garantice la viabilidad y la sostenibilidad partiendo de un mismo punto. Tampoco podemos asumir el pago de deudas históricas de la misma caja y, como condición previa ineludible, debe ponerse dinero encima de la mesa para empezar la discusión. 
No obstante, del análisis de situación del problema que nos ocupa no se pueden excluir otras variables que, en mi opinión, han incidido e inciden de forma notoria en este desajuste del modelo, que hoy todos o casi todos aceptamos como evidente. Efectivamente, los cambios tecnológicos, las mejoras retributivas del personal en no pocas autonomías, o el crecimiento del gasto farmacéutico, por poner algunos ejemplos, han tenido y tienen mucho que ver con estos desajustes. Es preciso caminar hacia un modelo retributivo con los mayores signos comunes posibles, y alejarnos de experimentos que quiebren el principio de confianza, porque así se sientan las primeras bases de las desigualdades del sistema vigente.

Habida cuenta de que los servicios sanitarios se financian con impuestos (complementados con el fondo de suficiencia) deberíamos entender que el actual modelo estaba encaminado a cubrir necesidades asistenciales del conjunto mínimo de prestaciones homogéneas en todo el Estado, dejando para cada comunidad el gasto suplementario de los servicios adicionales ofrecidos a los ciudadanos.

Con estos requisitos de partida para la reflexión, la cuestión a resolver es como debe financiarse el gasto. Para ello es necesario, en primer lugar definir, con la dificultad que ello conlleva, la capacidad que tenemos para mejorar la eficiencia en el gasto. Nuestra capacidad de intervención en este aspecto es limitada si analizamos el comportamiento histórico de las distintas variables que lo componen desde el punto de vista de la estructura presupuestaria de cualquier comunidad. Y cuando digo limitada, admito que hay un espacio para la mejora. Ese espacio para la mejora podemos obtenerlo en algunos aspectos del gasto farmacéutico, en la gestión personal o en los sistemas de contratación.

Pero es evidente que, con ello, no paliamos la insuficiencia financiera del sistema. La evolución de los ingresos no permite garantizar con el actual modelo su sostenibilidad. Se hace necesaria, por tanto, la inyección de mas recursos que den satisfacción a las necesidades.

En la reforma del modelo se deben tener en cuenta, además del factor población y, dentro de él, los mayores de 65 años, ya previsto en la fórmula actual, otros como la dispersión, y determinadas condiciones de orografía y situaciones especiales que configuran un sistema complejo en el que, en muchos casos, no existen las ratios adecuadas. Entenderán, por tanto, que una solución a un problema que tanto preocupa a los ciudadanos exige, además del compromiso político firme para afrontarlo, como de forma reciente se ha plasmado en la Conferencia de Presidentes, la necesidad de aunar esfuerzos en la misma dirección. Ahora más que nunca un pacto de Estado se convierte en una herramienta válida para afrontar el futuro con más garantías, en el que todos debemos comprometer- 
nos y buscar un fin común: la sostenibilidad de un sistema apreciado por los ciudadanos. Tenemos la garantía de que este debate se ha elevado al máximo nivel posible gracias a la implicación del Gobierno de José Luis Rodríguez Zapatero, pero tampoco podemos eludir nuestra responsabilidad y nuestro punto de vista privilegiado en una discusión en la que debemos fijar sus bases. El análisis riguroso de la realidad ofrecerá las claves exactas para transformar una apuesta por una solución. Entonces estaremos capacitados para ofrecer el máximo rendimiento en un sistema que, por la actitud inconsciente de otros, parece que no atraviesa su mejor momento. Resulta un tanto sorprendente y demagógico que ahora se exijan debates que, durante seis años, estuvieron ocultos para dejar crecer las ramas del problema, como el gasto farmacéutico. No quiero caer en la crítica fácil y cómoda, ejercicio que dejo para otros con más interés, pero tampoco puedo ocultar el malestar cuando se pretende reventar, de forma sistemática el foro de encuentro pactado entre las autonomías. Asturias exige respeto para su voz y para su posición, a veces inaudible en este marco cuando el baile de los representantes públicos se fija más en cuestiones mediáticas que en el verdadero interés que se deposita en estas citas.

Tenemos la oportunidad de romper esta tendencia, pero sólo la voluntad por alcanzar puntos de encuentros puede ser más fuerte que la debilidad por aprovecharse de estas circunstancias. El Gobierno al que representa tiene decidido que acudirá a cuantos foros sea necesario para sostener este discurso, basado en la racionalidad y que lucha por asumir un equilibrio con el que todos, y sin excepción, debemos estar comprometidos. El tiempo pasa de forma inexorable, y las soluciones empiezan a perder su efecto porque la teoría del desgaste también resulta dañina.

El Consejo Interterritorial tiene su papel, al igual que el Consejo de Política Fiscal y Financiera, pero admitamos el rango superior político de la Conferencia de Presidentes, por cierto, el precedente político más importante en nuestro país para vertebrar el Estado de las Autonomías.

Solo a modo de ejemplo del compromiso antes descrito, ya existe un acuerdo para que este año se debata el modelo que permita ofrecer las suficientes condiciones de estabilidad, que comenzarán a plasmarse en 2006. Así se construye un modelo que atiende a las necesidades de todos sobre criterios racionales, que permite que un ciudadano disfrute de las mismas garantías independientemente de su lugar de procedencia. Algo tan básico que venía reflejado en la Ley General de Sanidad de 1986 del malogrado Ernest Lluch, y que hoy vuelve a tomar el mismo vigor de entonces por la necesidad de abrir el debate que nos ocupa. 


\section{Financiación y futuro, claves para un equilibrio}

Reflexiones estas que nos deben obligar a todos a entender que la unión de nuestras posturas es esencial para conseguir los mejores resultados para los ciudadanos.

Antes indicaba que el Sistema Nacional de Salud es un patrimonio colectivo que hoy disfrutamos gracias a las conquistas sociales de las generaciones que nos precedieron; nuestra responsabilidad hoy es asegurar que este patrimonio llegará a las generaciones que nos sucedan, al menos, en las mismas condiciones de estabilidad con las que lo recibimos. Es una tarea de todos que estas reflexiones no se queden en meras palabras.

Decía Benjamín Franklin que "amaba la casa en la cual no veía nada superfino y encontraba todo lo necesario». En esta casa nuestra que es España, hemos abierto recientemente un debate apasionante, de alcance histórico, sobre la forma de culminar y perfeccionar algo tan sustancial y querido por todos nosotros: el modelo de Estado que hemos venido construyendo en este cuarto de siglo de andadura democrática.

La primera certeza es que el actual modelo de Estado ha pasado de ser una posibilidad apenas esbozada en el texto constitucional a una realidad que comparten 17 comunidades autónomas y que tiene forma jurídica y sustancia competencial, administrativa y financiera.

Sobre la cuestión de la financiación también es preciso separar con claridad el ruido que producen ciertas declaraciones de las nueces. Cuando se dice que hay que abordar la financiación general con el criterio de que «se pague por renta y se reciba por población» es necesario que se matice exactamente qué se quiere decir con este enunciado porque, de hecho, con el actual sistema ya se paga por renta y se recibe por población.

El 94\% del fondo general se reparte según la población, el 4,2\% a la superficie, el $1,2 \%$ a la dispersión y el $0,6 \%$ a la insularidad. En el sistema vigente existen también factores correctores que permiten, entre otras cosas, el acceso a los servicios públicos a ciudadanos que habitan en lugares de difícil acceso con la misma calidad y prestaciones que los que viven en grandes ciudades. Esta es una cuestión de gran importancia para preservar la igualdad de todos en comunidades autónomas que, como la asturiana, son especialmente montañosas y han sufrido históricamente las consecuencias económicas de tal circunstancia.

El fondo de la sanidad ya incluido en el sistema general establece también que la población protegida significa el $75 \%$, pero con una corrección que tiene en cuenta a la población mayor de 65 años, que cuenta en un $24,5 \%$. Es una corrección normal para una comunidad envejecida produce un gran consumo y es preciso proporcionar recursos al sistema para poder atenderlo. ¿Significa «pagar por renta y recibir por población» eliminar ese factor de corrección dirigido a la población mayor 


\section{Rafael Sariego García}

de 65 años? Creo sinceramente que sería una injusticia y rompería el principio de equidad establecido.

Lo cierto es que el modelo actual de financiación en España se puso en marcha hace menos de 3 años y, cuando logramos con él la unanimidad de las comunidades autónomas de régimen común, es porque entendíamos que tenía más elementos positivos que negativos. Asturias fue, precisamente, la última comunidad autónoma en firmar el acuerdo y, cuando lo hicimos, sopesamos todos esos factores, no sólo desde la perspectiva de la conveniencia propia, sino también desde la responsabilidad de dotar al conjunto del Estado de un modelo aceptable y duradero. Antes de cambiar o alterar el sistema pensamos que sería necesario retocarlo en aquellos aspectos que pudieran fallar y, sobre todo, conocer las posibilidades reales de su reforma. Porque, para cambiar un sistema de financiación de reciente creación, son necesarios muchos recursos que puedan ponerse sobre la mesa y, francamente, no veo ese fondo de recursos ni en las expectativas financieras actuales de España, ni en la evolución prevista del presupuesto pactado en el cuadro macroeconómico de la legislatura.

En cambio, tenemos que abordar de forma rápida y prioritaria la parte de financiación que específicamente corresponde a la sanidad. En todas las comunidades se puede observar una línea de tendencia de gasto sanitario que va creciendo de forma importante, y una línea de ingresos que no crece en la misma proporción.

Debemos afrontar de forma urgente la mejora de la eficiencia en aspectos como el gasto farmacéutico, la gestión de personal o los sistemas de contratación, y mantener un control sobre la evolución de un sistema que, por su complejidad y su importancia, no puede crecer desordenadamente.

Tenemos que incidir también en la evolución de los ingresos porque ya liemos utilizado todos los mecanismos a nuestra disposición y, en muchas comunidades autónomas, ya hemos puesto en marcha, el impuesto sobre hidrocarburos. Pero no es suficiente. En Asturias, por ejemplo, el gasto sanitario representa ya el $35 \%$ del total de nuestro presupuesto.

El sistema sanitario no es un servicio público cualquiera. Se partía de una conquista social irrenunciable, vital para los ciudadanos. Es un patrimonio colectivo generado con el esfuerzo de todos los españoles, que hoy es comúnmente aceptado como un buen sistema. Pero debemos reforzar su sostenibilidad minorando el ritmo de crecimiento del gasto y, simultáneamente, inyectando más ingresos hasta alcanzar los niveles medios europeos, de los que aún diferimos en un 3,5\% sobre el PTB. 
Lograr un Pacto de Estado para alcanzar una financiación estable, que permita sostener y mejorar el sistema sanitario, ha de ser, en mi opinión, el primero y más urgente de los retos que debemos afrontar para facilitar la cohesión social de esta España plural. Una España que, consciente de su diversidad, debe demostrar también su capacidad para concebir, de forma, corresponsable con el Estado, sus problemas comunes. Sería un enorme error caer en la tentación de buscar soluciones basadas en relaciones bilaterales, de las que tanto se abusó en otros tiempos. Tenemos ahora la oportunidad de demostrar que, tal y como decirnos con frecuencia, las comunidades autónomas también somos Estado y, por tanto, en la solución de sus problemas, desde foros multilaterales, también debemos estar todos.

\section{La salud en Asturias, una visión rápida}

La salud es el pilar básico del desarrollo humano, de su bienestar y de la calidad de vida de las personas. Sin embargo la noción de salud encierra tantos matices que buscar una definición precisa se antoja un ejercicio delicado y escurridizo. Debemos fijar una idea que va más allá de la ausencia de enfermedad. Se asienta sobre un modelo de salud que se pregunta cómo se encuentran sus gentes, sus calles, cómo de sanos están sus ríos y sus playas, cómo respiran sus fábricas o cómo laten sus carreteras.

Las autoridades políticas y sanitarias tenemos la obligación ética y legal de perseverar en el empeño de proteger la salud de sus ciudadanos en todos sus ámbitos. Este compromiso precisa hacerse explícito considerando, a todos los efectos, la salud como una prioridad de las políticas públicas. Y también, en la misma línea, garantizar los mecanismos que hagan eficiente la correcta gestión económica de los bienes.

El nuevo entorno económico y social de Asturias así como la aparición de nuevos problemas de salud constituye un escenario que si bien ofrece oportunidades de desarrollo y progreso social es al mismo tiempo fuente de amenazas a la sostenibilidad de nuestro sistema de protección de la salud tal y como lo concebimos hoy en día. Los cambios demográficos en nuestra población con un progresivo envejecimiento de la misma, la mayor prevalencia de enfermos crónicos, las crecientes demandas sociales y la aparición de nuevas tecnologías son sólo algunos de los retos en un futuro muy cercano.

Sin embargo invertir en la salud de los asturianos no es sólo una cuestión de ajuste en los balances económicos, implica también disponer de un instrumento de solidaridad y de redistribución social de la riqueza. El 
sector salud no debería ser entendido exclusivamente como causa de gasto sino como elemento generador de empleo (4,5\% del existente en Asturias), de fijación de la población a su entorno, de igualdad de oportunidades, etcétera.

La Ley 14/1986, de 25 de abril, General de Sanidad, establece los principios para la formulación de la política sanitaria de las Comunidades Autónomas a través de los planes de salud. En 1989 España se adhiere a la estrategia «Salud para Todos en el año 2000» de la OMS y sus objetivos fueron incorporados mayoritariamente por los planes de salud autonómicos. En nuestra Comunidad la Ley del Servicio de Salud del Principado de Asturias (Ley 1/92 de 2 de julio de 1992) asignó a la entonces Consejería de Servicios Sociales la elaboración del proyecto del Plan de Salud como principal instrumento de planificación sanitaria.

La materialización de la transferencia de las competencias sanitarias del Insalud desde el 1 de enero de 2002 al Principado de Asturias supone un decisivo cambio en la articulación del compromiso de acercar el núcleo de toma de decisiones a los ciudadanos/as y responsabilizarnos de nuestro sistema de salud y de nuestro futuro.

\section{La salud como prioridad política}

La noción de salud encierra tantos matices que buscar una definición precisa constituye un ejercicio delicado y escurridizo, probablemente condenado al fracaso. Si bien se renuncia a buscar una definición operativa para los conceptos de salud y enfermedad, no se oculta desde el principio su apuesta por una concepción global y positiva, una idea que va más allá de la ausencia de enfermedad. Nuestro compromiso se asienta sobre un modelo de salud que se pregunta cómo se encuentran sus gentes, sus calles, cómo de sanos están sus ríos y sus playas, cómo respiran sus fábricas y cómo laten sus carreteras. Se acepta sin ambivalencias el reto de considerar y profundizar en otros aspectos diferentes al ámbito exclusivamente sanitario.

La dimensión y amplitud del marco que se propugna para el desarrollo de las políticas de salud está en consonancia con el Plan de Salud para Asturias de 1992. Al igual que entonces, esta declaración implica poner en primera línea la perspectiva de los ciudadanos y aumentar nuestra sensibilidad a las necesidades y valores de las personas. Implica por otra parte asumir la capacidad de la salud pública y de las ciencias sociales para mejorar las condiciones de vida de las personas de forma que la elección más saludable sea la más fácil de adoptar. 
Financiación y futuro, claves para un equilibrio

Nuestro sistema sanitario es de carácter universal, accesible y de alta calidad, por lo que la inversión en salud se convierte en un instrumento de solidaridad y de redistribución social de la riqueza. Pilares básicos que nos obligan a unificar esfuerzos y caminar en la misma dirección. El resultado final será el fruto de las distintas aportaciones. En Asturias tenemos asumido que la dirección a tomar debe pasar por un debate abierto, sincero y transparente, alejado de otros intereses que enturbien las relaciones y próximo a una resolución apropiada para la magnitud del problema de la financiación. Entonces sí estaremos en condiciones de preservar aquellas bases por las que tanto hemos luchado y por asegurar principios tan importantes como los recogidos en la Ley General de Sanidad de 1986, en la que se consagró el derecho a la atención universal sea cual fuere la condición humana del usuario. Este es el camino que nos marcaron nuestros predecesores; esa nuestra obligación, no borrar sus huellas y preservar sus principales valores. 